\title{
Automated Detecting System for Elevator Guide Rails Based on Tilt Sensors and Acceleration Sensors
}

\author{
Yifeng Yang ${ }^{1}$, Wei Guo ${ }^{2}$, Yujun Wang ${ }^{2}$ and Wenxiu $\mathrm{Lu}^{3, *}$ \\ ${ }^{1}$ Department of Mechanical Engineering, Tsinghua University, Beijing, China \\ ${ }^{2}$ Hubei Special Equipment Inspection and Testing Institute, Wuhan, China \\ ${ }^{3}$ Department of Mechanical Engineering, Tsinghua University, Beijing, China \\ *Corresponding author
}

\begin{abstract}
Straightness, verticality and step height deviation are the main parameters to evaluate the safe and stable operation of the elevator. One kind of automated detecting system is devised to detect these multiple parameters of elevator guide rails at the same time exactly and efficiently. The detecting system, which is powered by a traction machine, is based on tilt sensors and acceleration sensors. The detecting system can verify the installation of elevator guide rails and the detection efficiency can be greatly improved.
\end{abstract}

Keywords—elevator guide rails; straightness; verticality; step deviation

\section{INTRODUCTION}

The Straightness, verticality and step height deviation of the elevator guide are the most important parameters that affect the operation of the elevator. The verticality error of elevator guide rail is one of the main factors that cause vibration and acceleration wear of elevator cab, and straightness error directly affects the comfort of elevator operation. The step deviation affects the stability of elevator operation.

The traditional measurement techniques for elevator guide rails are based on hand tools. The inspectors use feeler and guide rule to measure the deviation between the guide rails and a plumb line. In recent years, with the rapid development of the elevator industry, new methods to detect elevator guide rails efficiently become increasingly concerned. Some methods are proposed by many researchers. Liu and Chen [1] designed a system combined by an automatic-climbing robot and a laser sensor to detecting the guide rails. Laser sensor is accurate enough, but the volume of the robot is too larger for some scenarios. Noguchi, Naoaki [2] used an acceleration sensor, which was installed on a platform connected to the elevator cabs, to analyze the step deviation. The data acquired by the sensor can be dealt with to figure out the deviation. The system cannot be used before the installation of elevator cab because of without the power system. Liu [3] used a tilt sensor to measure the verticality of the guide rail. The system used long time step to measure the verticality. Long time step method is more convenient than continuously sampling, but has lower accuracy at the same time. An [4] compared several methods and devices, then the limits of different methods are pointed out in the paper. In paper [5-6], a method based on vibration analysis is put forward; a model of platform was designed to describe vibration. Some technologies including neural network and computer vision are applied to detect the elevator guide rails, some positive result has been gotten [7-8]

The single error of the elevator guide can be detected only by the methods above. If all the error of the elevator need to be detected, it is very difficult to replace the different measuring devices, which is time-consuming. This paper proposed an automated detecting system based on tilt sensors and acceleration sensors. The precision, sensitivity and stability in this automated detecting system are better than traditional measurement technique. The measurements of multiple parameters such as straightness, verticality and step height deviation of the elevator guide are fully automated one time.

\section{PRINCIPLE AND STRUCTURE}

\section{A. Structure of System}

As shown in Figure I, the structure of the whole system consists of three parts: traction system, measurement platform and base station. The measurement platform is driven by a traction machine, which can avoid the vibration and noise of motor in the measurement platform. The control system receives the signal transmitted by the base station, and then controls the measure platform to complete the detecting task. The speed of the platform is on-line controllable, and the design improves the accuracy.

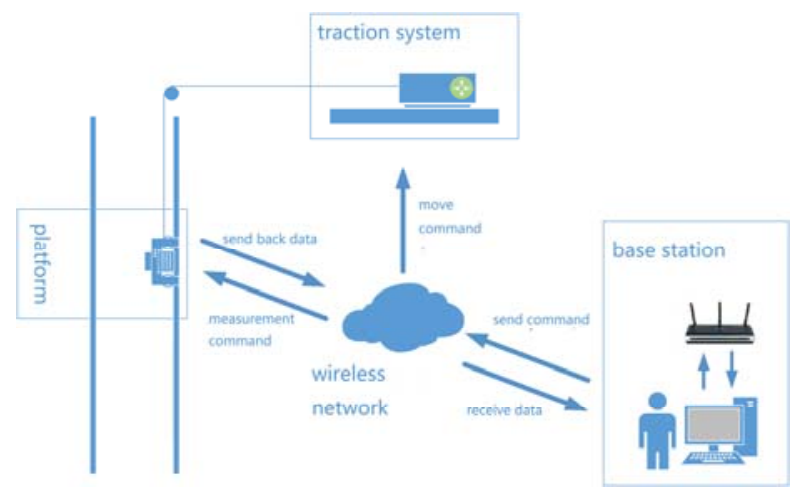

FIGURE I. STRUCTURE OF WHOLE SYSTEM

\section{B. Measurement Platform}

The measurement platform carries a SCM-based control system. One guide rail has three work surfaces, as shown in Figure II, including two side guiding surfaces and one top 
surface. Two tilt sensors and one acceleration sensor are assigned to each work surface.

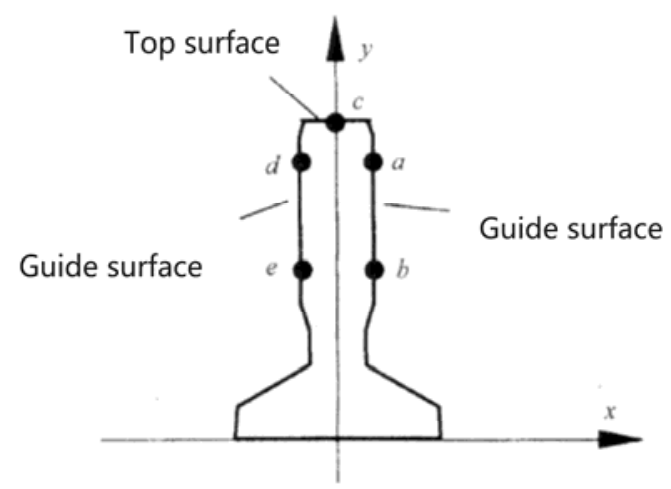

FIGURE II. WORK SURFACES OF GUIDE RAIL

Tilt sensors measure the angle in order to detecting the straightness and verticality of guide rails. Acceleration sensors measure the vibration of platform, the data can be processed to detect the step deviation at the joints. The system is shown in the Figure III.
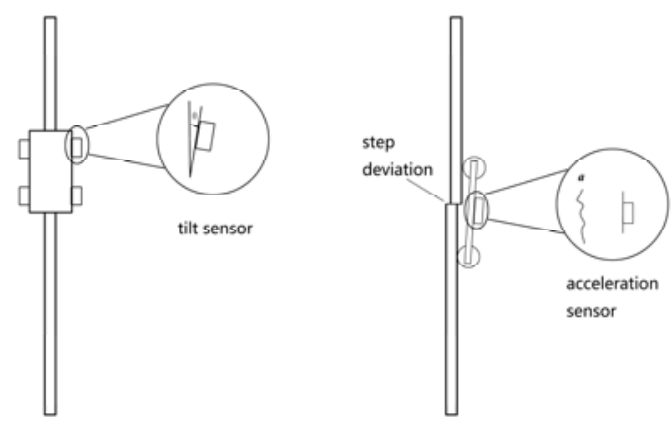

FIGURE III. WORK SURFACES OF GUIDE RAIL

The width of guide surface or guide rails is range from $10 \mathrm{~mm}$ to $36 \mathrm{~mm}$.A adaptive platform is designed to apply to different types of guide rails. The electromagnetic wheels installed on the platform located the platform on the guide surface. The magnetic intensities can be controlled in order to adapt to different working conditions. If the guide surface is smooth, user can raise the magnetic force to prevent derailment. The platform is shown in Figure IV.
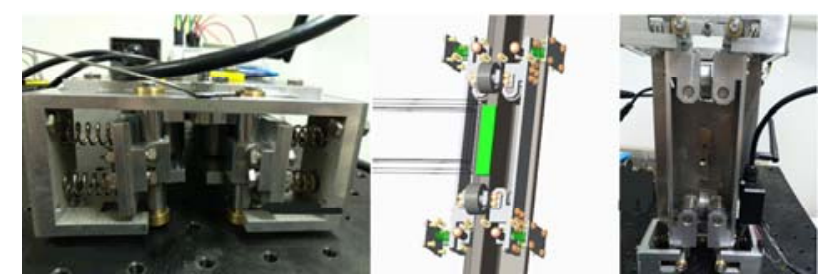

FIGURE IV. MEASUREMENT PLATFORM

There are three kind of acceleration sensor to be chosen, liquid tilt sensor, solid tilt sensor, mems acceleration sensor.
Liquid tilt sensor is chosen for the system because the liquid tilt sensor has damping so that it can filter high frequency noise. The sensor can measure the tilt angle of two axes. The accuracy of the sensor is $0.01^{\circ}$.

The data from the sensors is transmitted to the base station through the wireless transceiver system. The height of elevator guide rails can range from $10 \mathrm{~m}$ to $100 \mathrm{~m}$, The commonly used wireless module is based on $2.4 \mathrm{Ghz}$. The transmission distance of $2.4 \mathrm{Ghz}$ module is shorter than $433 \mathrm{Mhz}$ module, when they work in same power. The $433 \mathrm{Mhz}$ wireless module is chosen for the system. The transmission distance of the wireless transceiver system is $1000 \mathrm{~m}$.

\section{Base Station System}

Base station system consists of a computer and a wireless module. Relevant software is developed in the compute to control the traction system and to deal with the data measured by the sensors. Users can real-time monitor the state of the measurement platform, in case of colliding and derailment. The software will deal with the data to get the deviation of the three surfaces of guide rail, and then display the result as curve on the screen of the computer in real time. The data and the result will be stored in the disk for future reviewing.

\section{Base station system is shown in Figure V.}

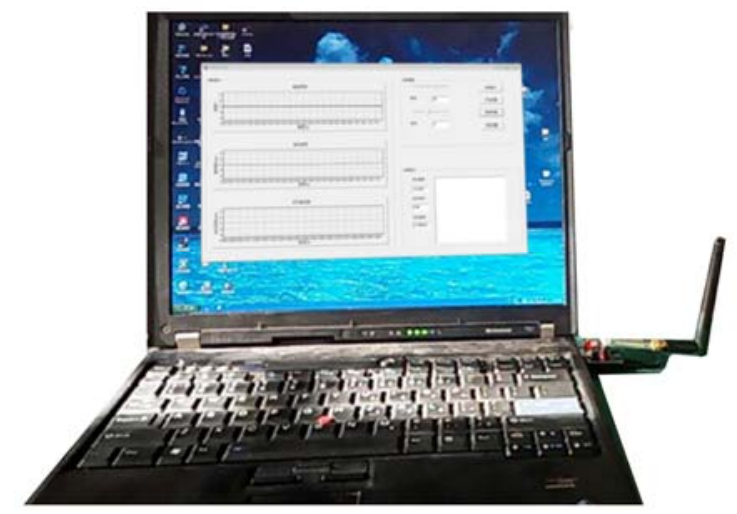

FIGURE V. BASE STATION

\section{Software System}

The software system consists of three parts: control software, data processing software and the embedded software.

The control software is installed in the compute of the base station system. The function of the software is to control and monitor the platform. There are two interfaces in the software, the one is to monitor the platform, the other one is to control the traction system.

The data processing software is used to deal with the data submitted by the sensors. And the results will be shown on the interface as curve. A method based on wavelet analysis is applied in the software to eliminate the noise. The precision of the result is higher than traditional methods.

The embedded software is installed in the SCM-based control system. The software will communicate with the base station to control the platform. 
Figure VI shows the interface of the software.

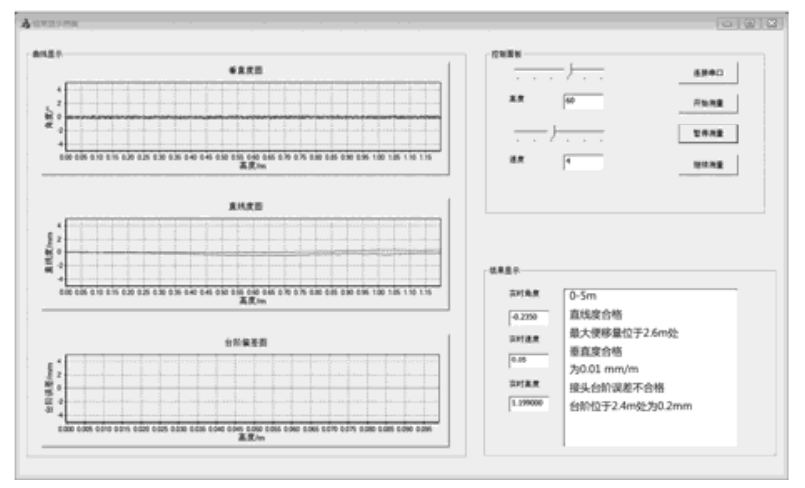

FIGURE VI. SOFTWARE INTERFACE

\section{EXPERIMENT}

Several experiments were conducted to test the whole system. A self-leveling laser collimator is used to calibrate the system. And then the tools stipulated in the China standard including guiding rule and feeler are used to get result as control group. Our automated system has a higher accuracy than traditional methods. Figure VII shows the straightness measurement value of different methods.

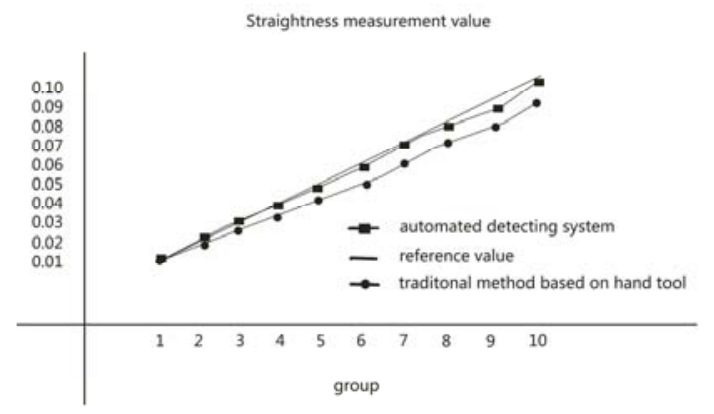

FIGURE VII. STRAIGHTNESS MEASUREMENT VALUE

\section{SUMMARY}

This system realized automated measurement of straightness, verticality, step deviation, of elevator guide rails. The accuracy of the result is better than previous methods. The system simplified the detecting process. In the future, more signal processing methods will be applied to improve the accuracy, and the platform will be redesigned to simplify structure and reduce the size. High-speed wireless module could possibly raise stability of the whole system.

\section{ACKNOWLEDGEMENT}

This research was financially supported by the National Science Foundation of China (Grant No. 51335006).

\section{REFERENCES}

[1] Wenang Liu, $\mathrm{Li}$ Chen, $\mathrm{Xu}$ zhiliang, The design of automatic straightening machine system for high precision elevator guide rails, Proceedings of the 33rd Chinese Control Conference, 2014, pp.2964-2968.
[2] Noguchi, Naoaki, Identification method of elevator rail deformation using operational acceleration data, Japan Society of Mechanical Engineers, 2012.

[3] Liu zhidong, Research on the perpendicularity detection robot of elevator guide based on tilt angle detection, Hebei University of Technology, 2011.

[4] Hongyun An, Study on the measurement method and experiment of the straightness of the elevator guide, Beijing Jiaotong University, 2008.

[5] Qianyin Zhang, The development of the inspection system for the straightness and distortion of the elevator guide, China Plant Engineering, 2017, Vol 4.

[6] Zhou Lei, Modeling, simulation and experiment for straightening lateral bending of elevator guide rail, Journal of Central South University, 2011 pp.1636-1642.

[7] Yu Qin, Jingjing Wu, Wei An, Meijuan Zhang, Research on automatic measurement system of elevator guide rail based on binocular vision, Modern Manufacturing Engineering , 2017, No 6.

[8] Kai Wang, Baoyu Wang, Chuancheng Yang, Calculation of elevator guide straightening based on Neural Network, Machinery Design \& Manufacture, 2012. 